\title{
KARL W. BUTZER Y LA LECTURA AMBIENTAL DE LA EXPULSIÓN DE LOS MORISCOS ${ }^{1}$
}

\author{
PABLO GIMÉNEZ FONT \\ Universidad de Alicante
}

Fecha de recepción: septiembre 2008

Fecha de aceptación: diciembre 2008

La multiplicación de estudios sobre los moriscos en las últimas décadas, junto con el avance en el conocimiento de la trayectoria de comunidades rurales antes y después de la expulsión de 1609, refuerza la idea de que este pueblo sigue ofreciendo una serie de temas abiertos y un buen número de cuestiones por dilucidar. A la revisión historiográfica se une la necesidad de incrementar el número de análisis de detalle que refuercen hipótesis más generales, con el fin de responder a preguntas complejas que proceden, de forma creciente, de planteamientos trasversales y enfoques más interdisciplinarios. A partir de esta idea, a la dimensión social y económica que alcanzó su presencia y expulsión en amplios territorios, hay que unir nuevas lecturas de tipo ambiental que pueden enriquecer ampliamente la investigación. Se trata de planteamientos de largo recorrido que incluyen capítulos como el protagonizado por el poblamiento islámico en la Península Ibérica -y los moriscos, como sus últimos representantes- dentro de la milenaria historia del paisaje mediterráneo. La complejidad de estas cuestiones y el riesgo de llegar a conclusiones lineales o simplistas, requiere de análisis críticos y de una auténtica transdisciplinariedad, de la cual los estudios dirigidos o realizados por Karl W. Butzer (Alemania, 1934) representan uno de sus máximos exponentes. A pesar

1. Este trabajo se inscribe en el proyecto de investigación titulado Los paisajes de la agricultura en España. Paisajes mediterráneos y canarios. Ref. SEJ2006-15331-CO2-02 (IP. Joan Tort). El autor quiere agradecer a los doctores Juan A. Marco Molina, Mauro S. Hernández y Joan F. Mateu Bellés las apreciaciones realizadas al texto original. 
de ello, sus conclusiones son todavía poco conocidas entre historiadores medievalistas y modernistas, también entre geógrafos y antropólogos.

\section{INTRODUCCIÓN}

Karl W. Butzer es un prestigioso científico con una reconocida trayectoria universitaria en Europa y, fundamentalmente, en EEUU, donde ha ejercido docencia en las universidades de Wisconsin-Madison, Chicago y Austin-Texas. De formación inicialmente geográfica, su producción científica es un ejemplo de contacto disciplinar entre las ciencias de la tierra (geomorfología, sedimentología, paleoedafología) y las ciencias sociales, de las que utiliza planteamientos provenientes de la arqueología, la historia, la antropología o la sociología. De ahí que, de forma genérica, pueda resumirse su vasto recorrido científico como un estudio de las interrelaciones dinámicas entre el medio ambiente y el ser humano, partiendo de las sociedades prehistóricas (desde los australopitecos africanos a la aparición del Homo sapiens sapiens en parte de este continente) hasta las más recientes (p. e. los siglos XIX y XX en Australia). Con esta idea, ha desarrollado diferentes campañas de trabajo de campo en Egipto, Nubia, Jordania, Este de África, Sudáfrica, Namibia, México, Australia, Chipre y España. En este último país ha trabajado en Mallorca y Cataluña (1959-72), Torralba-Ambrona (década de 1960, 1980-81) y en la Serra d'Espadà (Castelló de la Plana, entre 1980-87)².

Durante más de cincuenta años, su trayectoria investigadora y docente ha tenido, fundamentalmente, una reorientación metodológica dirigida a la aplicación de los estudios de Cuaternario y geomorfología en propuestas interdisciplinares de cronologías más recientes. De esta forma, su experiencia como cuaternarista se integró en un ambicioso planteamiento geoarqueológico que incorporaba metodologías procedentes de ciencias de la tierra como la ecología o la geografía física en combinación con otras ciencias sociales, como la antropología, la economía o la sociología ${ }^{3}$. Esta nueva concepción quedó reflejada, globalmente, en el importante manual de tipo teórico y

2. La prolongada y variada trayectoria del profesor Butzer únicamente nos permite presentar algunas pinceladas generales. Una sustanciosa autobiografía puede encontrarse en BUTZER, K.W.: «Les yeux ouverts», en Gould, P., y BAilly, A.: Mémoires de Géographes, Paris, 2000, pp. 43-77 (hemos utilizado la traducción francesa de Geographical Voices, New York, 2000). Igualmente un curriculum vitae bien desarrollado, con sus principales aportaciones, líneas de investigación y menciones honoríficas (fundamentalmente procedentes de los campos de la Geografía, la Geología y la Arqueología) puede encontrarse en https://webspace.utexas.edu/butzerkw/www/ (Universidad de Austin-Texas). Por último, son especialmente recomendables los textos realizados por geógrafos colaboradores de la Universitat de València: RossELLÓ, V. M.: «Entrevista con el Dr. Karl W. Butzer», en Debats, Valencia, 1987, n² 21, pp. 25-29; Más recientemente, Mateu, J. F.: Prólogo a la edición castellana de Butzer, K.W., Arqueología, una ecología del hombre. Método y teoría para un enfoque contextual, Barcelona, 2007, p. 13-21.

3. Dos ejemplos significativos de esta concepción global e interdisciplinar quedan reflejados en la publicación, con pocos años de diferencia, de los libros Geomorphology from the Earth (1976) y, como editor, Dimensions of Human Geography (1978). Su aportación más reciente al respecto puede verse en «Challenges for a cross-disciplinary geoarchaeology: The intersection between environmental history and geomorphology», Geomorphology, Amsterdam, 2008, nº 101, pp. 402-411. 
metodológico Archaeology as human ecology $(1982)^{4}$,, libro que recogía casi una década de investigación geoarqueológica en Oriente próximo y África y que sirvió de base para desarrollar proyectos en la línea de la ecología humana y de las aportaciones de la geografía a la arqueología. Investigaciones posteriores se dirigieron, de nuevo, hacia el mundo Mediterráneo (España, Grecia, Delta del Nilo, Chipre) y alguna de sus particularidades, como el antiguo e intenso poblamiento, los problemas de erosión de suelos, las estrategias de sustentabilidad, los usos del suelo relacionados con el regadío o la ganadería y la tradición agronómica de origen clásico e islámico. Aspectos dispares, pero relacionados, que han permitido construir un modelo inicial histórico-ecológico de los complejos agrosistemas mediterráneos ${ }^{5}$.

En este proceso de comprensión, la experiencia del proyecto de investigación llevado a cabo en la Serra d'Espadà durante los años 1980-1987, permitió verificar y modificar algunos esquemas en los que la conducta cultural y colectiva tenían un papel determinante en muchas de las relaciones causa-efecto que explican la historia del paisaje.

Con estas bases, el presente artículo pretende revisar la aportación de Butzer y su equipo al papel de la expulsión de los moriscos en la historia del paisaje de la Serra d'Espadà, llamando la atención sobre las posibilidades de aplicar hipótesis de trabajo similares al resto de antiguas zonas de montaña con poblamiento morisco. De esta forma, se propone un análisis bajo una perspectiva poco conocida en la historiografía española: la de la lectura ambiental de un colectivo que, dadas sus características intrínsecas (pautas culturales) y externas (marginación), mantuvieron un sistema de gestión algo diferente al de los cristianos que los sustituyeron.

4. BUtZER, K.W.: Archaeology as Human Ecology. New York: Cambridge University Press, 1982. La obra fue traducida al español en 1989 por la editorial Bellaterra. Aquí hemos utilizado la segunda edición de la misma, titulada: Arqueología, una ecología del hombre. Método y teoría para un enfoque contextual, Barcelona, 2007, 418 pp.

5. Sin ser exhaustivos, destacamos trabajos recientes de síntesis como BUTZER, K.W.: «Enviromental history in the Mediterranean world: cross-disciplinay investigation of cause-and-effect for degradation and soil erosion», Journal Archaeological Science, Amsterdam, 2005, n 32, pp. 1.773-1.800; BUTZER, K.W.: «Geoarchaeology, climate change, sustainability: a Mediterranean perspective», Prepared for Geoarchaeology 06 Conference, Exeter, 2006 (en prensa, cortesía de J. F. Mateu). A partir de la experiencia española, en la década de 1990 profundizó, junto a E.K. Butzer y otros colaboradores, en el estudio del regadío y la ganadería hispana con el objetivo de analizar la introducción de tecnología española en el regadío y la transmisión, durante la época colonial, de modelos de explotación ganadera peninsular en el norte y centro de México. La exportación e implantación de un modelo de gestión mediterránea en Nueva España ha centrado los esfuerzos de su equipo de investigación con resultados muy relevantes. Vid. ButZer, K.W.: «Ecology in the Long View: Settlement Histories, Agrosystemic Strategies, and Ecological Performance», Journal of Field Archaeology, Boston, 1996, vol. 23, n² 2, pp. 141-150. 


\section{PERSPECTIVA HISTÓRICA Y ECOLOGÍA HUMANA EN EL MEDITERRÁNEO}

El estudio de los ecosistemas mediterráneos, transformados casi de forma coevolutiva por una prolongada ocupación humana, reviste una gran dificultad a la hora de establecer relaciones causa-efecto entre los cambios en los usos del suelo, la intensidad en el aprovechamiento de los recursos naturales, los cambios climáticos y la capacidad de respuesta de estos ecosistemas ${ }^{6}$. Para ello se requiere, desde las ciencias sociales, ciertas habilidades en la comprensión ecológica; mientras que, desde las ciencias de la tierra, en ocasiones se obvian aspectos básicos del comportamiento humano y procesos históricos esenciales para la comprensión del paisaje y la degradación o sustentabilidad en una escala temporal amplia. La complejidad de estas temáticas, sin embargo, hace que cada punto de vista complemente al resto y sean necesarias distintas perspectivas pero con objetivos comunes. Butzer asume esta tarea interdisciplinar bajo el paraguas de la ecología humana, una ciencia de frontera disciplinaria que permite incorporar distintos resultados bajo un enfoque explicativo común. Como reconoce el autor, la ecología humana o cultural contiene planteamientos válidos para plantas y animales que no valoran correctamente el papel de la cultura y la cognición humana ${ }^{7}$. Sin embargo, con la incorporación de la Teoría de los Sistemas, el enfoque ecológico ofrece planteamientos que se han demostrado muy ventajosos. En primer lugar, destaca el interés por la capacidad de adaptación de los individuos y las comunidades a las situaciones cambiantes $^{8}$, como un elemento relevante del sistema. Al respecto, entran en juego aspectos como las elecciones adaptativas y las iniciativas individuales y colectivas (p.e. a la hora de acometer estructuras de reparto del regadío), las estructuras institucionales (p.e. sometimiento a una estructura feudal), el agregado de conocimiento común (p.e. conocimiento agronómico local) y la cultura, que convierten en una labor muy compleja pronosticar o, ni tan siquiera, proveer de estudios a niveles globales con resultados convincentes. De ahí que las escalas sean determinantes a la hora de formular modelos de acción y conducta humana, y los puntos de vista diacrónicos y sincrónicos sean necesarios para entender y contextualizar mínimamente los sistemas humanos.

Focalizar una investigación en un estudio de caso, a modo de subsistema, donde un pequeño rango de variables puedan delimitarse correctamente, es asimilado en gran medida como un experimento de laboratorio. Los resultados obtenidos se contextuali-

6. Sin ser un planteamiento novedoso, en los últimos años han aparecido aportaciones muy relevantes al respecto, como las de GROVE, A.T., y RACKHAM, O.: The Nature of Mediterranean Europe. An Ecological History, New Haven, 2001, 384 pp.; Horden, P., y Purcell, N.: The Corrupting Sea. A study of Mediterranean history, Oxford, 2000, 761 pp.; BLONDEL, J.: «The 'Design' of Mediterranean Landscapes: A Millennial Story of Humans and Ecological Systems during the Historic Period», Human Ecology, New York, 2006, no 34, pp. 713-729.

7. Butzer, K. W.: «The Realm of Cultural-Human Ecology: Adaptation and Change in Historical Perspective» en BUTZER, B. L. (ed.): The Earth as transformed by human action, Cambridge, 1990, p. 685.

8. Vid. Hawley, A. H., Ecología Humana, Madrid, 1975, p. 44. La obra de Hawley (reediciones inglesas posteriores) será utilizada ocasionalmente por Butzer. 
zan en un orden superior, pero a su vez pueden redefinir ese orden preestablecido con aportaciones novedosas que pueden ofrecer luz sobre la compleja red de interacciones entre una comunidad y su medio ambiente, posiblemente inapreciables a escalas más generales. De esta forma, el análisis de una sociedad humana como un agente más del sistema morfogenético se define a partir de la gestión secular de los recursos, basada en la producción de alimentos (estrategias de dieta), la tecnología («ecociencia rural»), los acuerdos sociales (con sus opciones alternativas manejadas), y el mantenimiento y reproducción del sistema (equilibrios sociales). Todos estos elementos se ven, a su vez, sujetos a las restricciones y limitaciones ambientales derivadas de un medio dinámico y sensible a los propios impactos antropogénicos. Se trata de un esquema parecido al presentado en otros estudios recientes de historia del paisaje, donde los planteamientos metodológicos -en este caso, desde un punto de vista geográfico- conectan enteramente con las amplias perspectivas de Butzer: las fuerzas de cambio y transformación del paisaje están en los lugares, los tiempos y los seres humanos. Desde la geografía histórica o desde la ecología cultural, el problema del conocimiento histórico del paisaje en un largo recorrido temporal es el problema entre el hombre y la naturaleza ${ }^{9}$.

Dentro de esta complejidad, las ventajas de los estudios específicos sobre territorios reducidos, como si de un experimento de laboratorio se tratara, también pueden ser limitantes, ya que algunos procesos o resultados pueden ser válidos para una escala y no serlo para otras. De ahí que, centrándonos en el proyecto que explicaremos más adelante, Butzer utilice la experiencia de la Serra d'Espadà para elaborar lo que el mismo define como un «modelo informal». La compleja red de interacciones entre una sociedad rural (el pueblo de Aín, en este caso) y su medio ambiente se contextualiza en un orden superior, previamente construido y «minimamente útil para interpretaciones a mesoescala de comunidades agrícolas sedentarias $\rangle^{10}$ en una montaña media mediterránea. La posibilidad de realizar interpretaciones acertadas a varias escalas, como ya se acometió exitosamente con los sistemas de regadío ${ }^{11}$, permite comprender mejor las relaciones sociedad-medio en un área de transformación acelerada, como fue la Serra d'Espadà antes y después de la expulsión.

Sin estudios similares en España, los resultados del proyecto y la madurez de reflexiones posteriores han constituido un modelo explicativo sobre las crisis -en este caso, fundamentalmente humanas- que pueden desencadenar procesos de degradación

9. GIMÉNEZ-FONT, P.: Las transformaciones del paisaje valenciano en el siglo XVIII. Una perspectiva geográfica, València, 2008, p. 17. Vid. también las interesantes reflexiones de VECCHIO, B.: «Sulla conoscenza storica del paesaggio mediterraneo», en ARIAS, J. y FoURNEAU, F. (eds.): El paisaje mediterráneo. Le paysage méditerranéen. Il paesaggio mediterraneo, Granada, 1998, pp: 81-90.

10. BUTZER, K.W.: «The Realm of Cultural-Human Ecology....» p. 686. La traducción es mía.

11. Una de las principales aportaciones de Butzer y su equipo a la historia del regadío peninsular es la propuesta de una tipología tripartita de los sistemas de riego, diferenciados en micro-, meso- y macrosistemas. Vid. Butzer, K. W.; Butzer, E. K.; Miralles, I. y MAteU, J. F.: «Irrigation agrosystems in eastern Spain: Roman or Islamic origins?», Annals, Association of American Geographers, Washington, 1985, $\mathrm{n}^{\circ}$ 75, pp. 479-509. Existe una traducción al catalán: «L'origen dels sistemes de regadiu al País Valencià: romà o musulmà?», Afers, Catarroja, 1988-1989, nº 7, pp. 9-68. 
y de cambio en ambientes mediterráneos. Dentro de su excepcionalidad, los moriscos representan un capítulo más en la dilatada historia ambiental de este territorio.

\section{EL PROYECTO AÍN-SERRA D’ESPADÀ: ETNOHISTORIA ECOLÓGICA DE UNA PEQUEÑA COMUNIDAD DE MEDIA MONTAÑA MEDITERRÁNEA}

Entre 1980 y 1987, un equipo transdisciplinar dirigido por Karl W. y Elisabeth K. Butzer, junto con Joan F. Mateu Bellés, Ismael Miralles (Universitat de València) y otros investigadores españoles y estadounidenses, llevó a cabo un proyecto de investigación archivística, arqueológica, etnológica y geomorfológica sobre historia ecológica de la Serra d'Espadà (Provincia de Castelló). El ejemplo de este espacio de sierras silíceas y valles encajados, donde se alcanza una altitud máxima de 1.083 m s.n.m y precipitaciones medias de entre 550 y $700 \mathrm{~mm}$, fue asimilado como representativo de otros territorios ecológica y sociológicamente similares.

Técnicas geoarqueológicas y geomorfológicas permitieron analizar los registros pleistoceno y holoceno y establecer hipótesis sobre influencias de cambios climáticos (fundamentalmente para el primer caso) y de la acción antrópica, relevante desde aproximadamente el Calcolítico y la Edad del Bronce en algunos sectores. El ámbito de estudio más detallado se centró en la cuenca de la Rambla de Artana $\left(52 \mathrm{~km}^{2}\right)^{12}$, donde se analizaron los problemas de erosión del suelo y degradación ambiental, contextualizados en un problema mediterráneo de gran actualidad ${ }^{13}$. Los resultados de los análisis sedimentológicos y palinológicos demostraron la sucesión de distintas fases de erosión del suelo y degradación de la vegetación de la sierra en tiempos prehistóricos e históricos. De esta forma, desde las poblaciones del Eneolítico, de la Edad del Bronce o los íberos, pasando por romanos y musulmanes hasta la colonización cristiana de los siglos XIII y XIV, el estudio de los suelos de laderas y sedimentos aluviales demostró la

12. Sobre la denudación fría pleistocena y la erosión antrópica holocena de este sector, vid. BUTZER, K. W. y MATEU, J. F.: «Pleistocene versus Holocene: Geomorphological Change in a small but steep Watershed of Mediterranean Spain», en Geoarqueologia i Quaternari litoral. Memorial M.P. Fumanal, València, 1999, pp. 97-111, especialmente el cuadro resumen de la p. 107.

13. Butzer se ha mostrado crítico con alguno de los postulados asumidos por parte de la comunidad científica en torno a la degradación de la cuenca del Mediterráneo, a causa de la acción del hombre. Este autor reclama perspectivas intelectuales y temporales más amplias para un problema complejo excesivamente simplificado. Vid. «Enviromental history...» $\mathrm{y}$ «Geoarchaeology, climate change,...». Es interesante comparar las reseñas que Butzer realiza de MCNEILL, J. R.: The Mountains of the Mediterranean World: An Environmental History, 1994, New York, 423 pp.; en Annals of the Association of American Geographers, Washington, 1996, vol. 86(4), pp. 780-803; y la de GrovE, A. T. y RACKHAM, O.: The Nature of Mediterranean Europe... en Annals of the Association of American Geographers, Washington, 2003, vol. 93(2), pp. 494-498. En la misma línea, vid. las opiniones compartidas por RosSELLÓ, V.M.: «Sedimentos, ambiente, hombre» en FUMANAL, M. P. y BERNABEU, J. (eds.): Estudios sobre Cuaternario. Medios sedimentarios. Cambios ambientales. Hábitat humano, 1993, València, p. 12. En España, son imprescindibles las reflexiones, que incluyen un detallado estado de la cuestión a principios de 1990, de MATEU, J. F.: «Morfogénesis mediterránea en tiempos históricos: limitaciones de un debate geoarqueológico», en Estudios de Arqueología Ibérica y Romana. Homenaje a Enrique Pla Ballester, 1992, Valencia, pp. 671-686. 
sucesión de etapas erosivas pero, también, de otros momentos de recuperación y menor presión humana sobre la tierra o aplicación de criterios más eficaces de sustentabilidad. Los resultados, a modo de historias aluviales, se correlacionaron con estudios realizados en otras cuencas fluviales cercanas, que ofrecieron dataciones parecidas y, por tanto, un contexto temporal y espacial más amplio de colonización y erosión ${ }^{14}$.

Sin embargo, la principal y más interesante impronta de este espacio venía determinada por un poblamiento musulmán que, tras la conquista cristiana de siglo XIII, fue cada vez más marginal, hasta desembocar en la conversión forzada al cristianismo en el siglo XVI y la aparición de los moriscos. Este pueblo despertó en Butzer un gran interés por su condición de aculturación islámica (los considera una representación de hispanorromanos completamente arabizados) ${ }^{15}$ y su difícil convivencia en un mundo cristiano dominador, cinco siglos después de la colonización islámica de la sierra. La expulsión de los moriscos en 1609, como colofón de un proceso de marginación creciente de la población autóctona iniciado con la conquista cristiana, significó la casi total despoblación de la sierra y una desintensificación de la acción humana sobre el ecosistema. Pero también supuso el nacimiento de comunidades rurales bajo un nuevo orden social y una nueva intensificación de la presión antropogénica sobre los recursos naturales. El proceso de cambio, como demostraba la historia ambiental de la Serra d'Espadà, no era nuevo, ya que se podía reconocer la repetición de trayectorias cíclicas (incremento / ausencia de erosión) a lo largo de este territorio. Con todo, su estudio estaba lleno de oportunidades debido a la datación de un momento de inflexión, cercano a la actualidad, que marcaba el cambio entre culturas territoriales y experiencias ecológicas diferentes: riqueza de fuentes archivísticas, presencia de yacimientos arqueológicos y un mundo rural casi intacto, con estructuras sociales, sistemas de regadío, estrategias de cultivo y elementos del paisaje herederos de uno u otro momento. Para Butzer y su equipo, se trataba de una excelente ocasión para desarrollar y matizar un enfoque metodológico madurado en torno a la ecología humana, con herramientas provenientes de la antropología, la historia, la arqueología y la geografía física.

Con estas técnicas transdisciplinares, la contextualización histórica y ambiental de la Serra d'Espadà, ajustada desde época islámica, y el análisis evolutivo del paisaje agrario en la cuenca de la Rambla de Artana diferenciando entre dos grandes momentos culturales, el musulmán y el cristiano, permitió construir un marco de generalización

14. La cuenca mejor estudiada, siguiendo la línea de investigación iniciada por J. F. Mateu, fue la del Júcar. Vid. Butzer, K. W.; Mateu, J. F. y Miralles, I.: «Urban geo-archaeology in Medieval Alzira (Prov. Valencia, Spain)», Journal of Archaeological Science, Amsterdam, 1983, vol. 10, pp. 333-349. De los mismos autores, «Las crecidas medievales del río Júcar según el registro geo-arqueológico de Alzira», Cuadernos de Geografia, Valencia, 1983, no 32-33, pp. 311-330.

15. Butzer, K. W.; Butzer, E. K. y Mateu, J. F.: «Medieval Muslim Communities of the Sierra de Espadan, Kingdom of Valencia», Viator. Medieval and Renaissance Studies, Los Ángeles, 1986, $\mathrm{n}^{\circ} 17$, p. 345. También en Rosselló, V. M.: «Entrevista...» p. 27. 
innovador ${ }^{16}$. Sin embargo, la comprensión de la conducta ecológica de los habitantes de la Sierra a lo largo de los siglos requería de un estudio de caso, más detallado, donde tratar de verificar un modelo complejo de interrelaciones entre los procesos sociales y la historia ambiental.

La pequeña población de Aín centró un estudio de carácter antropológico destinado a comprender los valores culturales y la mentalidad de los habitantes de la sierra, cuya conducta ecológica dependía, en gran medida, de estructuras sociopolíticas estables, por encima incluso de razonamientos puramente económicos. Fundada antes de 1150, debido al tardío establecimiento islámico en Espadà, las implicaciones paisajísticas del poblamiento musulmán tuvieron una gran entidad (por ejemplo, en la formación de los espacios de regadío). No obstante, el impacto de perturbaciones significativas, desde una óptica ambiental, no comenzó hasta el siglo XIV, cuando el reino cristiano estaba consolidado y las comunidades islámicas se encontraron social y económicamente coaccionadas por los conquistadores. Desde 1242 (documento de rendición de la sierra), los habitantes de las comunidades rurales vieron progresivamente erosionados sus privilegios con el incremento de las demandas fiscales, la persecución religiosa, la represión de rebeliones periódicas y la marginación generalizada de una población cada vez más numerosa, empobrecida y extorsionada. La culminación de este proceso se produjo a lo largo del siglo XVI, con los intentos de evangelización, la rebelión de 1526 y el fantasma de la expulsión que, finalmente, de produjo en 1609. Butzer realiza un planteamiento contextual para explicar el comportamiento ecológico de dichas comunidades que, cada vez más desarticuladas, dirigieron sus estrategias conservacionistas de gestión del medio a una supervivencia a corto plazo, con un uso inadecuado de los recursos que ha quedado reflejado en los resultados de las excavaciones arqueológicas (análisis polínicos y antracológicos, registros de erosión pre y post-conquista) $\mathrm{y}$ en la documentación escrita ${ }^{17}$. Esta idea, que propone la superación de umbrales de equilibrio en el uso sostenible del territorio hacia finales del siglo XIV, se contrapone con las nuevas comunidades cristianas formadas tras la expulsión, que se desarrollaron en ausencia de coacciones estructurales y bajo una relativa estabilidad social.

Los años posteriores al extrañamiento se caracterizaron por una lenta y costosa repoblación, lo que redujo claramente la presión humana sobre el medio y facilitó la recuperación de la vegetación natural que, según una visura realizada en 1570, presentaban un alto grado de degradación. De esta manera, la expulsión representó un mecanismo radical de desintensificación, después de un periodo marcado por el deterioro ecológico, y el aluvionamiento acelerado que caracterizó los siglos anteriores cesó de forma ostensible. No obstante, a partir del último tercio del siglo XVII y a lo largo del siglo XVIII la población de Aín aumentó rápidamente y volvió a incrementar la presión

16. Vid. los primeros artículos relevantes provenientes de los resultados del proyecto en BUTZER, K. W.; Butzer, E. K. y Mateu, J. F.: «Medieval Muslim...», pp. 339-420. Butzer, K. W.; Butzer, E. K.; I. Miralles, I. y MateU, J. F.: «Una alquería islámica medieval de la sierra de Espadán», Boletín de la Sociedad Castellonense de Cultura, Castellón de La Plana, 1985, nº 61, pp. 305-365.

17. Especialmente BUTZER, K. W.: «Enviromental history in the Mediterranean world:...», pp. 1.790-1.794. 
sobre los recursos. Muchas laderas, parcialmente recuperadas a lo largo del siglo XVII, fueron deforestadas para la expansión de los cultivos de secano, pero se artificializaron mediante complejos sistemas de terrazas de cultivo que favorecieron la infiltración del agua y evitaron la erosión. En lo que Butzer ha caracterizado como el periodo de «un pueblo mediterráneo tradicional $\gg{ }^{18}$, entre 1700 y 1830 , este nuevo proceso de intensificación se produjo dentro de un cuadro de estabilidad social y adaptación productiva a las exigencias del mercado, muy diferente al que caracterizó la última época de dominio musulmán. Bajo el predominio de una gestión más sostenible de los recursos, se fue construyendo un paisaje agrícola típicamente mediterráneo, con un peso importante de la ganadería trashumante y una progresiva reducción de las áreas boscosas, aunque el crecimiento estaba limitado por las restricciones provenientes del propio régimen señorial (principalmente, cargas fiscales onerosas y dificultades de acceso a la propiedad de la tierra y los recursos). Durante el siglo XIX se produjeron grandes cambios de orden socioeconómico, con la implantación del régimen liberal, que permitió el acceso a gran parte de las tierras comunales y señoriales fundamentalmente dedicadas a pastos, y la expansión del cultivo de la viña, favorecido por la plaga de filoxera en los viñedos franceses. La intensificación en el cultivo estaba sometida a las fuerzas del mercado, y la llegada de la filoxera a Castelló, en la primera década de 1900, repercutió aún más en un empobrecimiento generalizado de la población, aumentando las diferencias económicas y la reducción demográfica que comenzó a aparecer en el último tercio del siglo XIX. Durante el siglo XX, especialmente a partir de su segunda mitad, el sistema de aprovechamiento tradicional se colapsó, la población se redujo drásticamente, se abandonó la explotación forestal y la ganadería y se simplificó la agricultura, que en la actualidad perdura mantenida a tiempo parcial.

Esta descripción, breve síntesis de una completa investigación empírica en un micro-escenario como Aín, era necesaria para entender las dinámicas de adaptación sociocultural y de cambio, con la reevaluación de las estrategias agrarias y demográficas por parte de la comunidad, aspectos que contienen unas implicaciones ecológicas evidentes. La información obtenida del estudio de un caso local se contextualizó en órdenes superiores de generalización, empezando por la propia sierra $\mathrm{y}$, en muchos aspectos, abarcando gran parte del mundo rural mediterráneo español ${ }^{19}$. En este sentido, aunque con la intensificación de los cultivos del siglo XIX se superó un determinado umbral de sustentabilidad proporcionado por la explotación orgánica tradicional de la

18. Es el título de un epígrafe: A Tradicional Mediterranean Village: 1700-1830, en BUTZER, K. W.: «The Realm...», p. 688.

19. Algunos autores ponen en duda, precisamente, el marco de generalización desarrollado a partir del estudio de Espadà y, en concreto, de Aín. Vid. NAREDO, J. M.: «Las raíces económico-financieras de la crisis ambiental: un tema tabú en nuestros tiempos», en VIDAL-BENEYTO, J. (ed.): Hacia una sociedad civil global, Madrid, pp. 533-576. TELLO, E.: «La petjada ecolòica del metabolisme social: una proposta metodològica per analitzar el paisatge com a humanització del territori», Manuscrits, Barcelona, 2004, $\mathrm{n}^{\circ} 22$, p. 66. También Tello, E. y GARrabou, R.: «La evolución histórica de los paisaje mediterráneos: algunos ejemplos y propuestas para su estudio», en PAÜL, V. y TORT, J. (eds.): Territorios, paisajes y lugares: Trabajos recientes de pensamiento geográfico, Cabrera de Mar, 2007, pp. 19-64. 
agricultura mediterránea, los síntomas de degradación no fueron tan determinantes en el declive de la población como las variaciones de los precios del mercado y la coyuntura socioeconómica desfavorable.

\section{LA EXPULSIÓN DE LOS MORISCOS EN UNA PERSPECTIVA DE LARGO RECORRIDO}

El ejemplo de Aín-Serra d'Espadà representa una historia ecológica de largo recorrido que todavía hoy se está escribiendo, pero contiene una lectura especialmente sugerente e inédita sobre la expulsión, que puede convertirse, con las correspondientes adiciones de estudios en esta línea, en un marco de generalización convenientemente matizado.

Para Butzer, el proceso represivo contra la comunidad morisca, cuya creciente intensidad desembocó en la expulsión, supone un excelente ejemplo para demostrar que el comportamiento ecológico responde a una serie de contextos y de coyunturas complejas. Esta idea aporta una visión más completa sobre las relaciones causa-efecto en el cambio ecológico, pero también supone reconsiderar discursos que no reconocen el papel fundamental del comportamiento humano y de su capacidad adaptativa. Ciertos estereotipos, cuando no teorías sociales consolidadas, desprecian o dan por sentado este factor de enorme complejidad. Bajo condiciones de presión y extorsión cada vez más extremas, ¿cuántas poblaciones moriscas dejaron de observar comportamientos comunitarios e individuales, dentro de unos límites de adaptación ecológica, destinados a proyectar la comunidad hacia el futuro? Las sociedades cristianas, por el contrario, disfrutaron de cierta estabilidad y de normas sociales sólidas y renovadas, que aseguraban su continuidad, dentro de las complicaciones del medio rural de la época. Más tarde, surgirían nuevos problemas (como por ejemplo, la llegada de la filoxera y la crisis del cultivo de la vid) y, de nuevo, la comunidad demostró una capacidad de respuesta adaptativa, que ha permitido que en la actualidad todavía exista como tal. En similares términos ocurre con el ecosistema mediterráneo y su capacidad de adaptación y de resistencia. Los ecosistemas mediterráneos son producto de una coevolución milenaria entre el medio y las actividades humanas, por lo que se requieren perspectivas temporales mucho más amplias y habilidades en la comprensión ecológica para interpretar correctamente la degradación ambiental. De hecho, la capacidad de renovación de la vegetación y los suelos mediterráneos es muy elevada y compleja, de forma que los umbrales teóricos de dicha degradación se empiezan a discutir en el seno de la comunidad científica. Butzer los pone en duda e interpreta ciertos impactos en el ambiente biótico como reversibles, dentro de un mantenimiento a largo plazo ${ }^{20}$. El uso tradicional de la tierra, un aglomerado de conocimiento y experiencias de cerca de diez milenios, está dirigido a la conservación y a la adaptación ecológica, con el fin genérico de minimizar la vulnerabilidad hacia ciertos aspectos nocivos, como la aridez estival o la presencia de suelos pobres. En la capacidad de renovación de estos últimos,

20. ButZER, K. W.: «Enviromental history in the Mediterranean world:...», p. 1.795. 
por ejemplo, considera que, para la agricultura, el suministro de abono procedente del ganado puede llegar a ser más determinante que el propio espesor edáfico, de manera que los usos del suelo y la complementariedad en el aprovechamiento de los recursos son aspectos esenciales para el entendimiento de los agrosistemas mediterráneos, principalmente sostenibles y favorecedores de una elevada biodiversidad ${ }^{21}$. A pesar de todo ello, pueden existir desequilibrios más o menos pronunciados de forma puntual, originados por el hombre y condicionados, en ocasiones, por variaciones del clima ${ }^{22}$.

En un balance de síntesis, la acción humana se puede concebir como una transformación acumulativa del medio caracterizada por la presencia de trayectorias casi cíclicas denominadas genéricamente como períodos de intensificación y de desintensificación, períodos de crecimiento y de estancamiento/decrecimiento que no pueden asimilarse exactamente a la intensidad de la presión ejercida sobre los recursos naturales. Al contrario, ambas categorizaciones tratan de resumir cuestiones más complejas que abarcan sistemas globales como, en este caso, el mundo Mediterráneo ${ }^{23}$. Los momentos de intensificación se caracterizan por ir ligados a un crecimiento sistémico, un período de integración económica y comercio interurbano, de expansión comercial y de crecimiento demográfico como, por ejemplo, el contemplado durante la Edad del Bronce en el Mediterráneo. Sin embargo, el surgimiento de conflictos y guerras, la inestabilidad social y la inseguridad, pueden interrumpir el crecimiento económico sostenido y precipitar una simplificación socioeconómica, crisis urbana (ruralización), abandono agrícola y descuido de normas sociales (pastoreo, talas y rozas descontroladas) que normalmente repercute en la aplicación de usos de la tierra poco sostenibles o destructivos. Por encima de anomalías climáticas, una inestabilidad social de grandes proporciones puede influir en una inestabilidad ambiental, tal y como nos representa el ejemplo de los moriscos: de forma localizada, como es el caso de muchas montañas valencianas, su expulsión representó la culminación de un período de desintensificación, previo a la intensificación que dichas tierras conocerían en los siglos XVIII y XIX.

En las zonas de poblamiento morisco, el estudio de las décadas previas a la expulsión, en los términos antropológicos que plantea Butzer para Espadà, es dificultoso

21. BUTZER, K. W.: «Ecology in the Long View...», pp. 141-143. La intensa humanización de esta encrucijada biogeográfica, convierte a la cuenca del Mediterráneo en uno de los puntos calientes de biodiversidad planetaria (biodiversity hotspots). Agradezco las reflexiones al respecto de J. A. Marco Molina: «Transformaciones y alteraciones de los paisajes vegetales: de la Amazonía a la cuenca del Mediterráneo», en Certificado en Alteración y Regeneración de Espacios Naturales, València: Universitat de València, 2007, pp. 59-77.

22. Sobre esta cuestión, me remito a unas recientes reflexiones generales del autor en BUTZER, K. W.: «Challenges for a cross-disciplinary geoarchaeology...». Un completo estudio de caso (Chipre) puede verse en BUTZER, K. W. y HARRIS, S.: «Geoarchaeological approches to the environmental history of Cyprus: explication and critical evaluation», Journal of Archaeological Science, Amsterdam, 2007, $\mathrm{n}^{\circ}$ 34, pp. 1.932-1.952.

23. BUTZER, K. W.: «Enviromental history in the Mediterranean world:...», pp. 1.776-1.777. 
por la propia naturaleza de las fuentes: ${ }^{24}$ la escasa documentación referida al comportamiento de las comunidades en estos momentos sólo permite, en la mayor parte de las ocasiones, lanzar planteamientos de tipo deductivo, que después pueden verse refrendados con estudios más amplios e interdisciplinares. Sin embargo, un enfoque «de largo recorrido», junto con el reciente avance de la historiografía, permite englobar a los moriscos y a su trágico final en un proceso más amplio, el del cambio cultural que en tierras valencianas comenzó en el siglo XIII y se alargó, con su presencia, hasta los inicios del siglo XVII. El cambio cultural que se produjo en España con el avance de la conquista cristiana durante la Edad Media, se ha convertido en un tema central dentro de la nueva arqueología medieval, que sigue un modelo general de poblamiento en al-Andalus (especialmente sobre las pautas de los asentamientos rurales) y los importantes cambios realizados por los conquistadores cristianos ${ }^{25}$. El cambio, progresivo, a largo plazo y a gran escala, supone la implantación de una nueva organización del espacio relacionada con organizaciones sociales y percepciones del medio diferentes. Sólo este hecho nos puede hacer pensar en unas consecuencias ecológicas a medio plazo. Pero Butzer, como en gran medida hace Glick, considera acertadamente que los cambios en las normas de gestión del territorio son relativos desde un punto de vista ambiental: la gestión de los bosques, la agricultura intensiva o el peso de la ganadería trashumante y local, son aspectos que superan los límites socioculturales y pertenecen al mundo rural mediterráneo, entendido como un complejo compendio de tradiciones y cultura, de estratos de conocimiento más o menos entrelazados o encardinados. Un sistema complejo que no aparece en los textos. Buena muestra de ello la encontramos, precisamente, en la conquista cristiana y la apropiación e integración de técnicas indígenas e islámicas (cultivos, regadío) en un sistema social y cultural radicalmente diferente $^{26}$. Con los resultados del proyecto de la Serra d'Espadà, se puso de manifiesto que los procesos de degradación estuvieron presentes entre los siglos XIV y XVI, y que tales evidencias de un uso insostenible del suelo no son tan patentes en los siglos previos a la conquista cristiana y en los posteriores a la expulsión de los moriscos. Como ya se ha dicho, todo esto representa una muestra inequívoca de que el comportamiento ecológico viene definido por contextos específicos, como el que englobó la trayectoria de las comunidades de la Serra a partir de la conquista cristiana. De ahí que estos tres siglos supongan un momento clave de trasferencia política y de cambio cultural, ambos

24. Vid. las reflexiones al respecto en VINCENT, B.: «Être morisque en Espagne au xvi siècle», en GRUZINSKI, S., y Watchel, N. (dirs): Le Nouveau Monde. Mondes nouveaux: l'expérience américaine, Paris, 1996, pp. 505-510. Existe una reciente traducción al castellano en un libro recopilatorio del mismo autor: $E l$ Río Morisco, Valencia, 2006, pp. 155-161.

25. Por su carácter sintético, es especialmente recomendable el reciente estudio de GLICK, T. F.: Paisajes de conquista. Cambio cultural y geográfico en la España medieval, València, 2007, 253 pp. (traducción de Josep Torró).

26. Butzer, K. W.: «The Islamic Traditions of Agroecology: Cross-cultural Experience, Ideas and Innovation», Ecumene: Journal of Environment, Culture, Meaning, Sevenoaks, 1994, nº 1, pp. 7-50. 
potentes factores de desintensificación en ciertos ambientes que, como las áreas de montaña, se convirtieron en los últimos reductos del mundo islámico.

Esta perspectiva, que acapara distintos discursos narrativos, desde el arqueológico y el histórico hasta el geográfico y el ecológico, puede representar un avance en la teorización del cambio cultural en la España medieval que alcanza, con la presencia de los moriscos, la Edad Moderna. Al amparo de estas ideas, el estudio de estas comunidades, como representantes relativamente fieles de pautas de asentamiento y prácticas agrarias medievales, puede aportar, especialmente en zonas de montaña con escasa población cristiana, nuevas ideas sobre la reciente historia ambiental de espacios con trayectorias sociales y ecológicas parecidas.

\section{RELEYENDO LA EXPULSIÓN: NUEVAS PERSPECTIVAS CUATROCIENTOS AÑOS DESPUÉS}

A raíz de las reflexiones anteriores, en muchos espacios los moriscos y su aciago final representaron un punto de inflexión, el final y el principio de una nueva sociedad, de una nueva etapa. Cabría preguntarse hasta qué punto este hecho significó también un cambio en el paisaje y en la relación de las nuevas comunidades con su medio ambiente.

A lo largo de varios siglos, los conquistadores cristianos fueron implantando una nueva organización social y territorial cuyas características pueden resumirse, de forma muy sintética y acorde con nuestro planteamiento, en la adaptación a las exigencias de un nuevo sistema productivo. La zonificación del medio rural (sogueos, mensurabilidad de las tierras), relacionada con las crecientes demandas fiscales, debe entenderse dentro de los cambios en la gestión de los recursos y el favorecimiento de algunos cultivos, como el cereal o, más tarde, la morera. Con el objetivo de destinar una parte importante de la producción agraria al mercado, se intensificó el aprovechamiento de las aguas y cambiaron los modelos de administración y organización de los sistemas de regadío, aspectos básicos en la comprensión de los asentamientos y los usos del suelo ${ }^{27}$. En este sentido, la gestión hidráulica, considerada por Glick como un marcador cultural de primer orden, ha recibido una atención preferente por parte de la nueva arqueología medieval. El desarrollo de la molinería, con un nuevo papel central en el reparto del agua, es una de las consecuencias más relevantes, junto a nuevos acuerdos institucionales que suplían, en muchas zonas, una gestión de carácter tribal. No obstante, en este último aspecto, existe un acuerdo generalizado en que amplias zonas mantuvieron, con escasas modificaciones, el funcionamiento de la agricultura y los artefactos hidráulicos musulmanes. En realidad, el regadío, con su base andalusí

27. Para la comprensión del regadío en zonas montañosas con poblamiento casi exclusivamente mudéjar y morisco, vid. los recientes trabajos de TORRÓ, J.: «Terrasses irrigades a les muntanyes valencianes. Les transformacions de la colonització cristiana», Afers, Catarroja, 2005, n 51, pp. 301-356; y «Vall de Laguar. Asentamientos, terrazas de cultivo e irrigación en las montañas del Sarq al-Andalus: un estudio local», en Recerques del Museo d'Alcoi, Alcoi, 2007, nº 16, pp. 151-182. 
y los cambios cristianos, se hallaba en la base de la agricultura mediterránea y en su trasfondo policultural ${ }^{28}$.

Como es obvio, las transformaciones fueron dispares en cuanto a la intensidad y trascendencia de las mismas. A pesar de la heterogeneidad de casos, relacionada con la presencia de poblamiento mixto, la inserción de ciertas áreas productivas en los principales circuitos comerciales o con procesos de aculturación más o menos importantes, la idea de permanencia de gran parte del paisaje agrario islámico - o de las bases estructurales del mismo- resulta una sólida hipótesis de trabajo para ciertos territorios moriscos como la montaña, su hábitat por excelencia en las últimas décadas.

Como indica Butzer, no existían diferencias importantes entre las cosechas, la ganadería caprina o la trama urbana del Aín del siglo XII y el Aín del siglo XVII. En la montaña valenciana, donde los moriscos mantenían una posición subordinada respecto a los cristianos dominadores, permanecieron sin excesiva hibridación formas de asentamiento disperso, prácticas agrarias y factores culturales muy similares a los presentes en la baja Edad Media. Todavía en pleno siglo XVI, del que ha llegado una rica información documental, el maltrecho y decadente pueblo morisco puede analizarse como el último vestigio, desustanciado, de muchas pautas culturales de al-Andalus, que tienen una implicación paisajística y ecológica de primer orden. La riqueza documental de este período y la perfecta datación de su ocaso, han abierto una prometedora línea de investigación que ha ido depurando discursos en las últimas décadas.

Si los años que precedieron y siguieron a la expulsión supusieron un punto máximo de desintensificación, los cambios que acontecieron a partir de los inicios del siglo XVII sentarían las bases del importante desarrollo de los siglos siguientes, donde se sustenta una parte del paisaje agrario heredado en la actualidad. Con todo, las consecuencias de la expulsión es un tema recurrente que, sin embargo, todavía requiere de más estudios de caso $^{29}$. Sin entrar en la posible extensión e intensificación de las relaciones feudales, cuestiones actualmente discutidas, los resultados de una larga tradición en estudios sobre el siglo XVII permiten centrar la cuestión, desde un punto de vista ambiental, en los siguientes términos:

28. Esta es la idea que prevalece para afrontar el estudio del regadío en la Serra d'Espadà. Vid. BUTZER K. W.; Butzer, E. K. y MATEU, J. F.: «Orígenes de la distribución intercomunitaria del agua en la Sierra de Espadán (País Valenciano)», en Rosselló, V. M. (ed.): Los paisajes del agua, Valencia, 1989, pp. 229-238. Posteriormente se han realizado algunas apreciaciones a las conclusiones de este trabajo en SELMA, S.: «Evolució des de l'època andalusí de l'espai agrari irrigat a la Vall de Veo (Serra d'Espadà, Castelló)», en IV Congreso de Arqueología Medieval Española, Alacant, 1993, vol. III, pp. 567-574.

29. Un estado de la cuestión puede verse en BENíTEZ, R.: «La historiografía morisca: aspectos socioeconómicos (consideraciones sobre el «modelo de decadencia» de Bernard Vincent)», Balance de la historiografía modernista 1973-2001. Actas del VI Coloquio de Metodología Histórica Aplicada, Santiago de Compostela, 2003, pp. 239-247. ARdit, M.: Els homes i la terra del País Valencià, Barcelona, 1993, 2 vols. BARrios, M. (ed.).: Historia del Reino de Granada, vol II La época morisca y la repoblación, Granada, 2000. Considero especialmente sugerente el estudio de CISCAR, E.: «Economía y fiscalidad en los señoríos «pobres» de la Casa de Gandía en la época de la expulsión de los moriscos», Revista de Historia Moderna, Alicante, 2006, n 24, pp.123-152. 
En primer lugar, el predominio del contrato enfitéutico mediante la figura del establiment, atenuó el minifundismo morisco y supuso un lento pero contundente impulso roturador relacionado con cultivos de orientación comercial, como el trigo y la vid. En muchos espacios de montaña, este hecho significó un impulso, e incluso el inicio, en la construcción de grandes sistemas de terrazas de cultivo, desarrolladas masivamente hasta el siglo XIX y con repercusiones morfológicas de primer orden. Como hemos visto en el caso de Aín, las terrazas organizan positivamente la escorrentía y favorecen la infiltración y la formación de suelo. Pero su crecimiento se realizó sobre antiguos espacios de monte, de dedicación forestal y ganadera, y por tanto significó un cambio radical en los usos del suelo y un incremento de la presión humana sobre recursos naturales como los bosques o los pastos, de extensión cada vez más reducida ${ }^{30}$.

Las pautas de sustitución de cultivos eran variables según lugares, pero, en general, producciones como la morera o la expansión de cultivos regados trajeron asociada la sobreexplotación de los recursos hídricos, en la línea de lo que venía ocurriendo desde la conquista cristiana medieval ${ }^{31}$.

Por último, se reconoce un cambio en las pautas de asentamiento humano con una simplificación de la red de poblamiento ${ }^{32}$. Las bases de una nueva sociedad, presentes en las nuevas cartas de población, estaban muy condicionadas por el mapa jurisdiccional, pero también se vieron influenciadas por el importante cambio demográfico acontecido en algunas zonas de montaña ${ }^{33}$. Las dificultades del medio y unas condiciones señoriales excesivamente duras hicieron fracasar proyectos repobladores y obligaron a rectificar las condiciones iniciales de numerosas cartas de población, muchas veces en sintonía con la calidad de las tierras de cultivo ${ }^{34}$.

30. Algunas reflexiones ampliadas al respecto pueden verse en GIMÉNEZ-FonT, P.: «Cultura territorial y cambio ambiental en la Valencia moderna. Una lectura en clave de Geografía Histórica», en PAÜL, V. y TORT, J. (eds.): Territorios, paisajes y lugares: Trabajos recientes de pensamiento geográfico, Cabrera de Mar, 2007, pp. 81-95; Sobre la importancia del establiment en Valencia, vid., GIL OlcINA, A.: «Declive y ocaso de la enfiteusis señorial valenciana», Agricultura y Sociedad, Madrid, 1988, nº 49, pp. 293-318.

31. Para ésta y gran parte de las demás cuestiones referidas aquí, es de obligada consulta la obra de TORRó, J.: «Arqueologia de la Conquesta. Registre material, substitució de poblaments i transformació del espai rural valencià (segles XIII-XIV)», en BARCELÓ, M., et al. (eds.): El feudalisme comptat i debatut. Formació i expansió del feudalisme catalá. Ed. Universitat de València. Museu d'Història de Catalunya, 2003, pp. 153-200. Igualmente interesante es el trabajo de TRILLO, C.: «El paisaje vegetal en la Granada Islámica y sus transformaciones tras la conquista castellana», Historia Agraria, Murcia, 1999, nº 17 , pp. 131-152.

32. HALPERIN, T.: Un conflicto nacional: moriscos y cristianos viejos en Valencia, Valencia, 1980, pp. 267276. También ToRró, J.: Poblament i espai rural. Transformacions històriques, València, 1990, especialmente pp. 103 y ss.

33. Ver los excelentes estudios de Pla Alberola, P.: La población del marquesado de Guadalest en el siglo XVII, Alicante, 1983, 218 pp.; y «Despoblación y repoblación. La crisis del XVII en el Cuartel de La Marina y Las Montañas», en Nadal, J.: Evolución demográfica bajo los Austrias, Alicante, 1991, pp. 195-239.

34. Vid. la síntesis de ARDIT, M.: Els homes i la terra..., pp. 209 y ss.; o el trabajo de CisCAR, E.: Moriscos, Nobles y Repobladores, València, 1993, especialmente pp. 177 y ss. 
Todo este conjunto de aspectos interrelacionados, en consonancia con los inicios de un largo y determinante proceso de intensificación, no dejan de conformar un planteamiento preliminar. A pesar de la riqueza documental y la prolífica tradición historiográfica en la cuestión morisca, todavía quedan muchos aspectos complejos por dilucidar, relativos a la organización del territorio y la interrelación de estas comunidades con el medio. Poco o nada se sabe de antiguos equilibrios entre algunas actividades propias de los espacios yermos, como la ganadería o la obtención de leñas, y de éstas con los espacios de cultivo y con la propia red de poblamiento ${ }^{35}$. En este sentido, cabe preguntarse si los moriscos de zonas montañosas, en su aislamiento y sometimiento, mantuvieron la trasterminancia y la organización de los espacios forestales, o la gestión de los recursos comunales, en parecidos términos a como se encontrarían en época andalusí. Por ejemplo, aportaciones recientes desde la arqueología medieval indican la presencia de conjuntos coherentes de fenómenos como el regadío que, debidamente analizados, suponen una contribución al conocimiento de los tipos de asentamiento y los diferentes usos del suelo, incluidos sus cambios. A pesar de las hibridaciones con las prácticas cristianas, la posibilidad de reconstruir el paisaje morisco y su conjunto de relaciones internas y dinámicas, abriría grandes posibilidades para el análisis de la historia de importantes ecosistemas como los de montaña media mediterránea.

La complejidad de casos no permite, por el momento, afrontar con eficacia balances comparativos. Pero los trabajos de Butzer y su equipo de colaboradores, desde su perspectiva de largo recorrido y su carácter transdisciplinar, plantean hipótesis muy amplias que pueden impulsar investigaciones en direcciones específicas. El marco contextual desarrollado hasta aquí, permite aproximarse a la dimensión de los cambios en el paisaje de montaña tras la expulsión, abriendo un debate sobre múltiples cuestiones relacionadas, como las que conciernen a la importancia de las comunidades y sus trayectorias históricas locales ${ }^{36}$. Las conclusiones de Butzer, en parte fruto de una intensa y prolífica vida académica, demuestran que una gestión sostenible o insostenible desde el punto de vista ecológico puede tener un componente estructural, como ocurre en gran parte de la historia ambiental del Mediterráneo. Los moriscos aumentaron progresivamente la presión sobre la tierra, pero bajo una creciente inestabilidad social que incrementó la vulnerabilidad del sistema, de por sí ambientalmente frágil, aunque con gran capacidad de recuperación. A partir de esta idea, relativa a las propias personas y a su contexto, se pueden incluir en el análisis los cambios culturales (procesos mentales, de orden social y de orden económico) y las diferentes pautas de asentamiento y organización del espacio relacionadas con el mismo.

35. B. Vincent ya planteaba la importancia de los problemas ecológicos derivados de la expulsión y la repoblación en VINCENT, B.: «Un modèle de decadénce: le royaume de Grenade dans le dernier tiers du XVIe siècle», en Actas de las I Jornadas de metodología aplicada a las Ciencias Históricas, vol. III, Historia Moderna, Santiago de Compostela, 1975, pp. 213-217. En este mismo sentido, vid. las reflexiones de TORRÓ, J.: «Arqueologia de la Conquesta...», especialmente las pp. 192-193.

36. BUTZER, K. W.: «Enviromental history in the Mediterranean world:...», pp. 1.796-1.798. 